\title{
Basic Concepts of Fuzzy Matrices
}

\author{
P. Priya, R. Ramya, S. Sangeetha, A. Saranya \\ Assistant Professor \\ Dhanalakshmi Srinivasan College of Arts and Science for Women Autonomus \\ Perambalur
}

\begin{abstract}
A fuzzy matrix may be a matrix with elements having values in the fuzzy interval". In this article, the unit interval[0,1] and the interval [-1,1] are called fuzzy intervals[3]. Fuzzy matrices occur within the modeling of assorted fuzzy systems, with products usually determined by the " $\max (\mathrm{min})$ "rule arising from fuzzy set theory. In this paper some comfortable conditions for convergence beneath " $\max (\mathrm{min})$ " product of the powers of a sq. fuzzy matrix and of a fuzzy state method square measure established.Convergence of powers of a fuzzy matrix original Research Article Journal of Mathematical Analysis and applications, volume 57, Issue 2, February 1977, pages 476-480 Michael. G. Thomason[4].
\end{abstract}

\section{INTRODUCTION:}

Fuzzy matrices were introduced for the first time by Thomason[4], who discussed the convergence of powers of fuzzy matrix. Fuzzy matrices play a vital role in scientific development. The basic and essential fuzzy matrix theory is given. Instead, the authors have solely tried to convey those essential essentially required to develop the fuzzy model. The authors don't gift elaborate mathematical theories to figure with fuzzy matrices; instead they need given solely the required properties by method of examples. Several authors have presented a number of results on the convergence of the power sequence of fuzzy matrices[2,5,8]. Ragab at al[1] presented some properties on determinant and adjoint of square fuzzy matrix. Kim and Roush[6] studied the canonical form of an idempotent matrix. Hashimoto [7] studied the canonical form of a transitive matrix. Kolodiejczyk [8] presented the canonical form of a strongly transitive matrix. $X$ in $[10,11]$ studied the controllable fuzzy matrix. Ragab et al.[12] presented some properties of the properties of the min-max composition of fuzzy matrices. Kim [13] presented some important results on determinant of a square fuzzy matrices. The new operators and some applications of fuzzy matrices are given in $[14,15,16,17]$. The authors feel that the book should mainly help social scientists who are interested in finding out ways to emancipate the society. In this section basic concepts about fuzzy matrices are introduced. Basic notions of matrices are given in section one in order to make the book self contained.

\subsection{TYPES OF FUZZY MATRICES :}

\subsubsection{INTRODUCTION :}

Just we recall the definition of fuzzy matrix. A fuzzy matrix may be a matrix that has its parts from [0,1]. We as in case of matrix have rectangular fuzzy matrix, fuzzy square matrix, fuzzy row matrix, diagonal matrix \& fuzzy column matrix. We just illustrate them by the following examples.

\subsubsection{DEFINITION AND EXAMPLES :}

\section{DEFINITION :1.1.2.1}

Consider a matrix $A=\left[a_{i j}\right]_{m \times n}$ wherea $a_{i j} \in[0,1], 1 \leq \mathrm{i} \leq \mathrm{m}$ and $1 \leq \mathrm{j} \leq \mathrm{n}$. Then $\mathrm{A}$ is a fuzzy matrix.

FUZZY ROW MATRIX :1.1.2.2

Let $\mathrm{A}=\left[\mathrm{a}_{1}, \mathrm{a}_{2}, \mathrm{a}_{3} \ldots \mathrm{a}_{\mathrm{n}}\right], \mathrm{a}_{\mathrm{ij}} \in[0,1] ; j=1,2, \ldots \mathrm{n}$. Then $\mathrm{A}$ is called a $1 \mathrm{xn}$ fuzzy row matrix or fuzzy row vector.

\section{Examples}

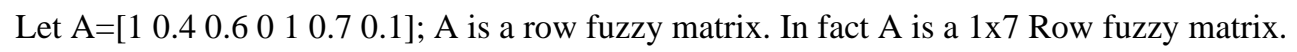

$A_{1}=[0,1]$ is a $1 \times 2$ row fuzzy matrix.

$\mathrm{A}_{2}=\left[\begin{array}{lllllll}1 & 0 & 1 & 0 & 0.6 & 0.2 & 110\end{array}\right]$ is a $1 \times 9$ row fuzzy matrix. 
FUZZY COLUMN MATRIX:1.1.2.3

Let B $=\left[\begin{array}{c}\boldsymbol{b}_{1} \\ \boldsymbol{b}_{2} \\ \vdots \\ \boldsymbol{b}_{\boldsymbol{m}}\end{array}\right]$

Where ai $€[0,1] ; \mathrm{i}=1,2, \ldots \mathrm{m}$. Then $\mathrm{A}$ is called a $\mathrm{mx} 1$ fuzzy column matrix.

\section{Examples:}

Let $B=\left[\begin{array}{c}1 \\ 0 \\ 0.14 \\ 0 \\ 1 \\ 1\end{array}\right]$

$\mathrm{B}$ is $6 \mathrm{x} 1$ column fuzzy matrix.

$$
\mathrm{B}_{1}=\left[\begin{array}{l}
\mathbf{1} \\
\mathbf{0}
\end{array}\right]
$$

is a $2 x 1$ column fuzzy matrix.

$$
\mathrm{B}_{2}=\left[\begin{array}{c}
0.06 \\
0.2 \\
0.14 \\
0 \\
1 \\
0.03 \\
0.12 \\
0.31 \\
0
\end{array}\right]
$$

is a $9 x 1$ column fuzzy matrix

\section{FUZZY SQUARE MATRIX:1.1.2.4}

$$
\text { Let } \mathrm{A}=\left[\begin{array}{cccccc}
a_{11} & a_{12} & \ldots & a_{1 j} & \ldots & a_{1 n} \\
a_{21} & a_{22} & \ldots & a_{2 j} & \ldots & a_{2 n} \\
\vdots & \vdots & \vdots & \vdots & \vdots & \vdots \\
a_{i 1} & a_{i 2} & \ldots & a_{i j} & \ldots & a_{i n} \\
\vdots & \vdots & \vdots & \vdots & \vdots & \vdots \\
a_{n 1} & a_{n 2} & \ldots & a_{n j} & \ldots & a_{n n}
\end{array}\right]
$$

wherea $_{\mathrm{ij}} \mathrm{E}[0,1], 1 \leq \mathrm{i}, \mathrm{j} \leq \mathrm{n}$.

Then A is a fuzzy square matrix.

\section{Example:}

Consider the fuzzy matrix

$$
\mathrm{A}=\left[\begin{array}{ccccc}
0 & 0.1 & 1 & 0.6 & 0.7 \\
0.4 & 1 & 0 & 1 & 0 \\
1 & 0.5 & 0.6 & 0.2 & 1 \\
0 & 1 & 0 & 0.1 & 0 \\
0.2 & 0.6 & 1 & 1 & 0.2
\end{array}\right] \text { is a square fuzzy matrix }
$$

InfactA is a $5 \times 5$ square fuzzy matrix. 


\section{Examples:}

Consider the fuzzy matrix

$$
B=\left[\begin{array}{lll}
1 & 0 & 0 \\
0 & 1 & 0 \\
0 & 0 & 1
\end{array}\right]
$$

$\mathrm{B}$ is a square $3 \times 3$ fuzzy matrix.

Consider the fuzzy matrix

$$
C=\left[\begin{array}{cc}
1 & 0.3 \\
0.1 & 0.04
\end{array}\right]
$$

$\mathrm{C}$ is a $2 \mathrm{x} 2$ fuzzy square matrix.

\section{FUZZY RECTANGULAR MATRIX:1.1.2.5}

Let $A=\left[a_{i j}\right]_{m x n}(m \neq n)$ where $a_{i j} \epsilon[0,1], 1 \leq i \leq m$ and $1 \leq j \leq n$. Then $A$ is a fuzzy rectangular matrix.

Examples:

Let $A=\left[\begin{array}{cccc}0 & 0.3 & 1 & 0.2 \\ 0.1 & 1 & 0 & 0.6\end{array}\right]$

$\mathrm{A}$ is a $2 \mathrm{x} 4$ fuzzy rectangular matrix.

Let $\quad \mathrm{T}=\left[\begin{array}{cc}0 & 1 \\ 0.3 & 0.1 \\ 1 & 1 \\ 0.8 & 0.71 \\ 0.5 & 0.11 \\ 0 & 0.1\end{array}\right]$

$\mathrm{T}$ is a $6 \mathrm{x} 2$ fuzzy rectangular matrix.

\section{FUZZY DIAGONAL MATRIX :1.1.2.6}

A fuzzy square matrix $A=\left[a_{i j}\right]_{n x n}$ is said to fuzzy diagonal matrix if $a_{i j}=0$ when $i \neq j$, where $a_{i j} \in[0,1], 1 \leq I, j \leq n$.

\section{Example :}

$\left[\begin{array}{ccc}\mathbf{0 . 4} & \mathbf{0} & \mathbf{0} \\ \mathbf{0} & \mathbf{0 . 3} & \mathbf{0} \\ \mathbf{0} & \mathbf{0} & \mathbf{0 . 9}\end{array}\right]$ is a fuzzy diagonal matrix of order 3

This square matrix is additionally denoted by $[0.4,0.3,0.9]$

FUZZY SCALAR MATRIX : 1.1.2.7

A fuzzy square matrix is claimed to be fuzzy diagonal matrix, if all its diagonal entries are equal.

Thus a fuzzy square matrix $A=[a i j] n$ xn is said to be a fuzzy scalar matrix if

Operations of $\{$ maximum|most"> most and minimum we have a tendency to shall outline the subsequent 3 operations on fuzzy matrices. $\left\{\begin{array}{l}\boldsymbol{a}_{\boldsymbol{i}}=\mathbf{0} \text { when } \boldsymbol{i} \neq \boldsymbol{j} \\ \boldsymbol{a}_{\boldsymbol{i j}}=\boldsymbol{\alpha} \text { when } \boldsymbol{i}=\boldsymbol{j}\end{array}\right.$ where $\alpha \in[0,1], 1 \leq \mathrm{i}, \mathrm{j} \leq \mathrm{n}$.

Example :

$[0.3]$ and $\left[\begin{array}{cc}\mathbf{0 . 4} & \mathbf{0} \\ \mathbf{0} & \mathbf{0 . 4}\end{array}\right]$ are fuzzy scalar matrices of order 1 and 2 respectively. 


\section{EQUALITY OF FUZZY MATRICES:1.1.2.8}

Two fuzzy matrices of the same type are said to be equal iff there elements in the corresponding positions are equal.

\subsection{OPERATION ON FUZZY MATRICES :}

\subsubsection{INTRODUCTION}

Operations of maximum and minimum we define the following three operations on fuzzy matrices.

a) Maximum of matrices

b) Minimum of a matrix by a scalar

c) Max min of matrices

\subsubsection{Operation I Maximum of matrices}

If 2 fuzzy matrices are of a similar kind, then they are said to be conformable for addition. But the question arises that after we add 2 fuzzy matrices, then the resultant matrix is not a fuzzy matrix .So incase of fuzzy matrices of same type, max operation is defined.

\section{DEFINITION :1.2.2.1}

Let $A=\left[a_{i j}\right]_{m \times n}$ and $B=\left[b_{i j}\right]_{m x n}$ be two fuzzy matrices. Then their sum, denoted by $A+B$ is defined as $A+B=\operatorname{Max}\{A, B\}$ ie., $\left[a_{\mathrm{ijj}}+\mathrm{b}_{\mathrm{ij}}\right]=\left[\operatorname{Max}\left(\mathrm{a}_{\mathrm{ij}}, \mathrm{b}_{\mathrm{ij}}\right)\right]_{\mathrm{mxn}}$ for $1 \leq \mathrm{i} \leq \mathrm{m}, \mathrm{i} \leq \mathrm{j} \leq \mathrm{n}$.

\section{Example :}

Let $A=\left[\begin{array}{ccc}0 & 0.3 & 0.9 \\ 0.4 & 0.3 & 0.1 \\ 1 & 0.8 & 0.4 \\ 0.5 & 0.2 & 0.6\end{array}\right]$ and

$$
\mathrm{B}=\left[\begin{array}{ccc}
0.6 & 0.7 & 0 \\
0.9 & 0.5 & 0.5 \\
0.7 & 1 & 0 \\
0.6 & 0.8 & 0.5
\end{array}\right]
$$

Then $\mathrm{A}+\mathrm{B}=\operatorname{Max}\{\mathrm{A}, \mathrm{B}\}$

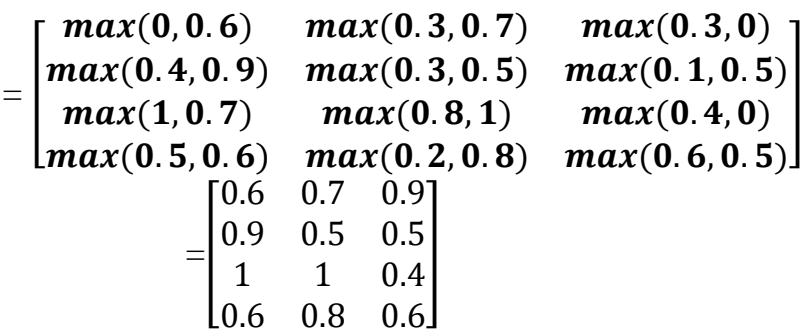

In a similar manner we are able to outline the distinction of 2 fuzzy matrices of same kind because the gamma hydroxybutyrate operation.

Thus just in case of fuzzy matrices of same kind,

$\mathrm{A}-\mathrm{B}=\operatorname{Max}\{\mathrm{A}, \mathrm{B}\}=\mathrm{A}+\mathrm{B}$ 
DEFINITON:1.2.3.1

Let $A=\left[a_{i j}\right]_{m x n}$ be any fuzzy matrix and $k C F$, where $F E[0,1]$ is a fuzzy unit interval. Then scalar multiple of $A$ by $k$, denoted by

$\mathrm{KA}$ or $\mathrm{AK}$ is given by

$\mathrm{KA}=\mathrm{AK}=\left[\mathrm{Ka}_{\mathrm{ij}}\right]_{\mathrm{mxn}}=\min \left[\left(\mathrm{k}, \mathrm{a}_{\mathrm{ij}}\right)\right]_{\mathrm{mxn} ; \mathrm{a} \mathrm{ij}} \in[0,1], 1 \leq \mathrm{i} \leq \mathrm{m}, 1 \leq \mathrm{j} \leq \mathrm{n}$.

Thus KA or AK is the matrix obtained when each entry of $\mathrm{A}$ is multiplied by $\mathrm{k}$.

\section{Example:}

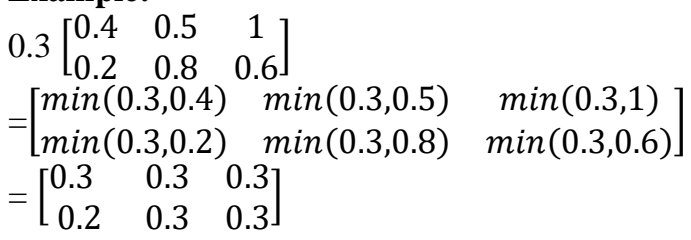

\subsubsection{OPERATION III MAX MIN OF MATRICES: \\ CONCLUSION}

In this paper we discuss about the concept of fuzzy matrices and the convergence of power of fuzzy matrices which helps in the field of scientific development .It is also essential in the development of fuzzy models

\section{REFERENCES}

[1] H. Hashimoto, canonical form of a transitive fuzzy matrix, fuzzy sets and systems, 11(1983),157-162.

[2] W. Kolodziejczyk, convergence of powers of s.transitive fuzzy matrices, fuzzy sets and systems; 26(1988), 127-130.

[3] L.J. Xin, controllable fuzzy matrices, fuzzy sets and systems,45(1992), 313-319.

[4] L.J. Xin, convergence of powers of controllable fuzzy matrices, fuzzy sets and systems, 62(1994), 83-88 .

[5] M.Z. Ragab and E.G. Emam, The determinant and adjoint of a square fuzzy matrix, fuzzy sets and systems, 61(1994),297-307.

[6] J.B. Kim, Determinant theory for fuzzy and Boolean matrices, CongressusNumerantium, (1988), 273-276

[7] A.K. Shyamal and M. Pal, Two new operators on fuzzy matrices, J.Applied mathematics and computing, $15(2004), 91-107$.

[8] A.K. Shyamal and M. Pal, Distance between fuzzy matrices its applications, ActasienciaIndica, XXXF.M(1)(2005),199-204.

[9] A.K. Shyamal and M. Pal, Distance between fuzzy matrices and its applications-I, J.Natural and physical sciences, 19(1)(2005), 39-58.

[10] A.K. Shyamal and M. Pal, Distance between intuitionstics fuzzy matrices, V.U.J. physical sciences, 8(2002), 81-91

[11] Zadeh,L.A., "Fuzzy sets", Information and control, 8(1965),338-353.

[12] M.G.Thomason, convergence of powers of a fuzzy matrix,J,mathAnal.Appl., 57(1977), 476-480.

[13] K.H.Kim and F.W.Roush, Generalised fuzzy matrices, fuzzy sets and systems, 4(1980),293-315.

[14] J.B.Kim, Determinant theory for fuzzy and Boolean matrices, congressusnumerantium, (1988), 273-276.

[15] H. Hashimoto, canonical form of a transitive fuzzy matrix, fuzzy sets and systems, 11(1983), 157-162.

[16] W. Kolodziejczyk, convergence of powers of s.transitive fuzzy matrices, fuzzy sets and systems; 26(1988),127-130.

[17] L.J. Xin, controllable fuzzy matrices, fuzzy sets and systems,45(1992), 313-319.

[18] L.J. Xin, convergence of powers of controllable fuzzy matrices, fuzzy sets and systems, 62(1994), 83-88

[19] M.Z. Ragab and E.G. Emam, The determinant and adjoint of a square fuzzy matrix, fuzzy sets and systems, 61(1994),297-307.

[20] J.B. Kim, Determinant theory for fuzzy and Boolean matrices, CongressusNumerantium, (1988), 273-276.

[21] A.K. Shyamal and M. Pal, Two new operators on fuzzy matrices, J.Applied mathematics and computing, 15(2004), 91-107.

[22] A.K. Shyamal and M. Pal, Distance between fuzzy matrices its applications, ActasienciaIndica, XXXF.M(1)(2005),199-204

[23] A.K. Shyamal and M. Pal, Distance between fuzzy matrices and its applications-I, J.Natural and physical sciences, 19(1)(2005), 39-58.

[24] A.K. Shyamal and M. Pal, Distance between intuitionstics fuzzy matrices, V.U.J. physical sciences, 8(2002), 81-91. 